\title{
Extensão Médica Acadêmica: um projeto da Faculdade de Medicina da Universidade de São Paulo para treinamento clínico e humanização do cuidado em saúde de alunos da medicina, nutrição e fisioterapia
}

\section{"Extensão Médica Acadêmica": healthcare humanization and clinical training of medicine, nutrition and physical therapy students from the School of Medicine of University of São Paulo}

\author{
Leandro Ryuchi luamoto', Mauro Shigueharu Oide Junior², Jéssica Tioma \\ Nakayama ${ }^{3}$, Regina Sayuri Yamashiro Shiotuki ${ }^{4}$, Juliana Mika Kato ${ }^{3}$, \\ Renato Kurebayashi ${ }^{5}$, Danilo Henrique da Silva Suzuki ${ }^{3}$, \\ Ariana Lee $^{6}$, Christian Valle Morinaga ${ }^{7}$
}

Iuamoto LR, Oide Junior MS, Nakayama JT, Shiotuki RSY, Kato JM, Kurebayashi R, Suzuki DHS, Lee A, Morinaga CV. Extensão médica acadêmica: um projeto da Faculdade de Medicina da Universidade de São Paulo para treinamento clínico e humanização do cuidado em saúde de alunos da medicina, nutrição e fisioterapia / "Extensão Médica Acadêmica": healthcare humanization and clinical training of medicine, nutrition and physical therapy students from the School of Medicine of University of São Paulo. Rev Med (São Paulo). 2012 jul.-set.;91(3):194-7.

RESUMO: A Extensão Médica Acadêmica (EMA) foi fundada em 1998 na FMUSP visando à formação de médicos que valorizam o exame clínico e o relacionamento humano. É um projeto de voluntariado atualmente organizado por estudantes de medicina, fisioterapia e nutrição da USP. O EMA é sustentado por três pilares: ensino, assistência e pesquisa. O projeto é realizado aos sábados em dois bairros carentes da cidade de São Paulo, e tem como objetivo oferecer um atendimento ambulatorial gratuito de qualidade, que priorize cuidados em saúde e humanização na relação médico-paciente. Os pacientes são atendidos por alunos e os casos são discutidos com profissionais de saúde, e durante a semana são realizadas reuniões com todos os membros do projeto, na Faculdade de Medicina da USP, contribuindo para a consolidação e aprofundamento dos conceitos em saúde. Este modelo de ensino complementa os estudos em sala de aula, pois permite o desenvolvimento de habilidades geralmente pouco exploradas durante o início da graduação tradicional. O EMA incentiva seus alunos a valorizarem a relação médico-paciente desde o primeiro ano da graduação. Assim, o projeto tem êxito em reunir pessoas dispostas a lidar com pacientes, aprender sobre saúde e ensinar outros estudantes. Como resultado, muitos de seus membros continuam a participar do projeto após o término da faculdade, tornando-se orientadores comprometidos a passar adiante o conhecimento adquirido durante sua prática profissional.

DESCRITORES: Humanização da assistência; Equipe de assistência ao paciente; Relações médico-paciente; Assistência à saúde; Programas voluntários.

1. Graduando em Medicina pela Faculdade de Medicina da Universidade de São Paulo e Vice-Presidente do Projeto EMA.

2. Graduando em Medicina pela Faculdade de Medicina da Universidade de São Paulo e Presidente do Projeto EMA.

3. Graduanda(o) em Medicina pela Faculdade de Medicina da Universidade de São Paulo e Diretor(a) do Projeto EMA.

4. Graduanda em Medicina pela Faculdade de Medicina da Universidade de São Paulo e Coordenadora de Pesquisa do Projeto EMA.

5. Graduando em Fisioterapia pela Universidade de São Paulo e Diretor do Projeto EMA

6. Graduanda em Nutrição pela Universidade de São Paulo e Diretora do Projeto EMA.

7. Médico do Departamento de Clínica Médica da Faculdade de Medicina da Universidade de São Paulo e Fundador do Projeto EMA.

Endereço para correspondência: Faculdade de Medicina da Universidade de São Paulo. Av. Dr. Arnaldo, 455 - Subsolo Pacaembu, São Paulo, SP. CEP: 01246-000. 
ABSTRACT: The Academic Medical Extension (EMA) is a volunteer project of the School of Medicine of University of São Paulo organized by students of Medicine, Physical Therapy and Nutrition of University of São Paulo. It was founded in 1998 in order to provide a better academic development to students who value physical examination and human relations, besides providing to students in the beginning of graduation an early contact with patients and promoting an exchange of information between those three areas of health. EMA is sustained by three pillars: assistance, education and research, thus constituting an alternative to community-based education and assistance with a focus on humanization. The project is carried in two regions in the city of São Paulo and aims to offer these needy populations a free ambulatory care with quality, promote health and prevention. On Saturdays, undergraduate students see the patients and discuss the clinical case with a health profes-

\section{INTRODUÇÃO}

Odesenvolvimento da ciência e da tecnologia em saúde, nos últimos anos, é certamente impressionante, todavia, apresenta severas limitações ${ }^{1,3}$. Pensando-se nisso, o projeto Extensão Médica Acadêmica (EMA) é fundado em 1998 na Faculdade de Medicina da Universidade de São Paulo (FMUSP) com a proposta de realizar atendimentos médicos com uma visão humanizada do paciente em que acadêmicos, junto com profissionais da saúde voluntários, pudessem prestar assistência e discutir formas de um melhor cuidado e atenção em saúde.

Criado pelo Dr. Ademar Chies e seis acadêmicos de medicina, dentre eles o Dr. Christian Valle Morinaga - atual responsável pelo projeto - com o apoio do Centro Acadêmico Oswaldo Cruz, o projeto surge como "Projeto Médico Fundação Julita", atuando em apenas duas salas da instituição filantrópica da Fundação Julita no bairro Jardim São Luiz, SP.

Ao longo dos anos, o projeto foi se expandindo tanto em tamanho, quanto em complexidade, área de abrangência e número de voluntários, passando a se chamar "Extensão Médica Acadêmica": Em 2002, com o apoio do Prof. Dr. Paulo Hilário do Nascimento Saldiva, foi firmado um acordo com o Laboratório Central do Hospital das Clínicas da FMUSP para o treinamento de membros do projeto na realização de coleta de exames laboratoriais. Em 2004, aprimorando a multidisciplinaridade e integralidade do atendimento, profissionais e acadêmicos da fisioterapia iniciam suas atividades na Fundação Julita. Em 2005, o Serviço de Assistência Social da Penha (SASP), instituição filantrópica do bairro da Penha (SP) cede espaço para nossa atuação. Em 2010, com um caráter intedisciplinar, a fisioterapia amplia seu campo de atuação através de atendimen- sional; once a week, they attend a meeting with their group, which includes students of the three areas of health. During this meeting, the students report the clinical case and are assisted by other students to conduct the case and teach what they've learnt about the patient, collaborating with the establishment and deepening of the concepts in health. The project seeks to encourage their students since the first year of college to value the physician-patient relationship. Thus, it brings together people willing to work without the intention to earn a profit, but to learn more about health and to teach other students. As a result, members still participate in the project after graduation, as doctors committed to pass on their experience and knowledge.

KEYWORDS: Humanization of assistance; Patient care team; Physician-patient relations; Delivery of health care; Voluntary programs.

tos também no SASP; e a nutrição passa a integrar o projeto. Atualmente, o projeto conta com cerca de 120 acadêmicos, abrangendo 250 atendimentos no ano de 2011.

\section{OBJETIVOS}

Apresentar o projeto EMA e discutir estratégias de humanização e interdisciplinaridade no atendimento aos pacientes e na formação acadêmica de alunos de áreas da saúde.

\section{Estratégias de atuação}

O EMA é um projeto de voluntariado sustentado por três pilares - ensino, assistência e pesquisa - e tem como objetivo oferecer um atendimento ambulatorial gratuito de qualidade para duas populações carentes nas zonas sul e leste de São Paulo.

O projeto valoriza o exame clínico, contato com o paciente e desenvolvimento de uma boa relação humana, pontos de grande importância nas práticas em saúde? .

Os atendimentos são realizados aos sábados pela manhã, das 8 horas às 14 horas, no Serviço de Assistência Social da Penha (Penha), e na Fundação Julita (Jardim São Luis).

O processo de aprendizado da humanização do cuidado vai além da sala de aula. Ele envolve aspectos complexos de política, treinamento filosófico, práticas de ensino e atitudes profissionais de saúde e educação $0^{4,5}$. Por conseguinte, com a finalidade de auxiliar na formação de profissionais mais humanizados na relação com o paciente, alunos desde o primeiro ano de graduação são estimulados conversarem e fazerem a história clínica dos pacientes, para que 
antes de aprenderem a fazer diagnósticos, aprendam conhecer a pessoa de forma mais completa e humana. Estes atendimentos ocorrem sem limite de tempo e, portanto, permitem ao aluno conversar adequadamente com o paciente e estabelecer uma relação de confiança com o mesmo, utilizando também a propedêutica e a técnica necessárias para um cuidado igualmente adequado.

No Serviço de Assistência Social da Penha os pacientes são atendidos por um a três alunos de medicina e nutrição, sendo que membros mais graduados e antigos no projeto passam seus conhecimentos aos mais novos, auxiliando-os na realização do atendimento. Após a consulta, os casos atendidos são discutidos com profissionais de cada área, de modo a que se chegue às melhores condutas para cada paciente. Esse modelo de atendimento é seguido nas consultas da fisioterapia, porém estes são realizados separadamente, haja vista que nem todos os pacientes necessitam desse serviço. Por esse motivo, os atendimentos fisioterapêuticos realizados no EMA ocorrem por meio de encaminhamento médico. $\mathrm{Na}$ Fundação Julita os atendimentos da medicina e fisioterapia ocorrem nos mesmos moldes, porém ainda não há atendimento nutricional, o que o projeto pretende incluir ainda no ano de 2012.

Os locais de atendimentos contam com farmácias próprias, abastecidas com medicamentos doados por empresas e pessoas da própria comunidade. Visando facilitar o acesso ao tratamento, 0 medicamento prescrito é cedido ao paciente sempre que possível e, sempre que necessário, coleta de sangue, fezes e urina são feitas no também no próprio local. As coletas são realizadas por membros do projeto, do curso de medicina, previamente treinados por técnicos de enfermagem do DLC-HCFMUSP (Divisão do Laboratório Central do Hospital das Clínicas da Faculdade de Medicina da Universidade de São Paulo), que também realiza a análise laboratorial das coletas obtidas. Durante as coletas o médico orientador do dia supervisiona os procedimentos adotados e também auxilia os alunos para a realização dos mesmos quando necessário.

Os membros do projeto são divididos em grupos numericamente homogêneos de aproximadamente 10 a 15 pessoas, denominados "panelas", de modo a garantir o acompanhamento longitudinal dos pacientes atendidos. Atualmente o SASP abrange uma panela constituída por alunos de fisioterapia e três constituídas por alunos de medicina e nutrição, e a Fundação Julita abrange duas panelas da fisioterapia e três da medicina. Por esse motivo, além dos atendimentos aos sábados, são realizadas reuniões semanais em cada panela, para que os alunos responsáveis pela consulta anterior repassem o caso atendido, contribuindo assim para o aprendizado, fixação e aprofundamento de conceitos em saúde. Este modelo de ensino complementa os estudos em sala de aula, pois permite o desenvolvimento de habilidades geralmente pouco exploradas durante o início da graduação tradicional dos cursos participantes.

Assim, com o objetivo de oferecer atendimentos ambulatoriais gratuitos de qualidade, o projeto integra ensino, assistência e pesquisa, possibilitando o levantamento de dados para o estudo epidemiológico das regiões atendidas e proporcionando aos acadêmicos um aprendizado baseado na prática clínica, através da realização de um atendimento voltado aos cuidados em saúde e à humanização na relação médico-paciente.

\section{RESULTADOS}

Em um levantamento feito em setembro de 2002, o projeto já havia realizado 729 atendimentos e feito 130 exames laboratoriais. Só no ano de 2011, foram feitos mais de 250 atendimentos e realizados quase 100 coletas de material para exames laboratoriais.

Nesses 14 anos de história, estima-se que houve mais de 2000 atendimentos e mais de 500 coletas para exames laboratoriais, o que significa que mais de 600 pacientes se beneficiaram com o projeto.

O EMA teve início com apenas um médico e seis acadêmicos, e atualmente conta com mais de vinte profissionais da área da saúde e aproximadamente 60 acadêmicos da medicina, 40 da fisioterapia e 20 da nutrição, o que mostra a evolução e consolidação do projeto como uma forma de extensão e ensino.

Muitos membros continuam a participar do projeto após o término da faculdade, tornando-se orientadores comprometidos em passar adiante o conhecimento adquirido durante sua prática profissional.

Os dados mais importantes, todavia, são subjetivos e podem em parte ser traduzidos pelo depoimento dos pacientes.

No final de 2011, a valorização da relação médico-paciente e a qualidade de atendimento foram relatadas em depoimentos de pacientes atendidos pelo projeto: "O carinho e a dedicação desses jovens é algo incomum", "Aqui o exame físico é muito bem feito, vocês olham da raiz do cabelo até a raiz das unhas".

A humanização é um tema em grande discussão na medicina e, os pacientes são enfáticos na importância do médico cuidador, capaz de ouvir seus problemas com carinho. Eles sabem que os alunos estão em fase de treinamento e não são vistos como 
pessoas sem domínio da técnica, mas sim como alunos preocupados com o cuidado e a qualidade do exame que realizam, o que é muito valorizado.

O EMA incentiva seus alunos a valorizarem a relação médico-paciente desde o primeiro ano da graduação. Assim, o projeto tem êxito em reunir pessoas dispostas a lidar com pacientes, aprender sobre saúde e ensinar outros estudantes.

Graças ao esforço de todos os membros, o EMA já foi classificado entre os 10 melhores projetos no II e V Prêmio Saúde Brasil, além de receber o prêmio como um dos melhores projetos de voluntariado na apresentação oral do Brazilian International Congress of Medical Students (Braincoms) 2012.

\section{DISCUSSÃO}

O EMA tem por objetivo ser um projeto de referência no desenvolvimento profissional e pessoal dos voluntários, no âmbito da humanização e bem estar social, através das atividades realizadas em grupos interdisciplinares e com a própria população.

Há necessidade de aprimorar a participação da medicina, fisioterapia e da nutrição no projeto de forma interdisciplinar, pois essa forma complexa de atendimento é um grande desafio, pois exige tempo, espaço e estrutura para possibilitar a interação entre os diversos alunos dos diferentes cursos. É importante que haja grande envolvimento dos alunos na diretoria, estimulando o desenvolvimento da humanização com a contribuição de diferentes visões.

É essencial, também, a promoção de reuniões interdisciplinares com a participação de todos

\section{REFERÊNCIAS}

1. Ayres JRCM. O cuidado, os modos de ser (do) humano e as práticas de saúde. Saúde Soc. 2004;13(3):16-27.

2. Ayres JRCM. Hermeneutics and humanization of the health practices. Cien Saúde Col. 2005;10(3):549-60.

3. Bettinelli LA, Waskievicz J, Erdmann. Humanização do cuidado no ambiente hospitalar. Mundo Saúde. 2003; 27(2):231-9. os alunos, eventualmente de outras áreas da saúde que não fazem parte do projeto, e principalmente de profissionais, para ter a oportunidade de promover o aperfeiçoamento clínico e a humanização na relação paciente - profissional de saúde.

Em longo prazo, o projeto visa introduzir outras áreas da saúde nos atendimentos, como a psicologia e a terapia ocupacional, que já sugeriram a sua incorporação. Para isso, faz-se necessário um planejamento das dinâmicas de atendimentos, sejam estes ambulatoriais ou campanhas pontuais, de modo que o cuidado médico permaneça como uma das questões centrais do projeto.

\section{CONCLUSÕES}

Caminhando na direção oposta a uma tendência de fragmentação do conhecimento, o EMA acredita que seja de suma importância que alunos de graduação nas áreas de saúde tenham, desde o primeiro ano, uma formação profissional que valorize um trabalho multidisciplinar voltado não somente ao tratamento da doença, mas também, ao projeto de vida dos pacientes, trabalhando em conjunto com os mesmos visando à melhoria de sua qualidade de vida.

Em relação ao aprendizado, além da prática clínica mais humanizada, o projeto promove e incentiva que os alunos conheçam mais profundamente outras áreas da saúde, o que certamente levará a uma formação diferenciada ao final de sua graduação, com um profissional dotado uma visão mais ampla de saúde e com maior habilidade para trabalhar com as diversas especialidades em saúde.

4. Casate JC, Correa AK. The humanization of care in the education of health professionals in undergraduate courses. Rev Esc Enferm USP. 2012;46(1):219-26.

5. Costa E, Borenstein MS. Problematizando para humanizar: uma proposta de transformação do cuidado em uma enfermaria psiquiátrica. Texto Contexto Enferm. 2004;13(1):163-70. 